\title{
Amenability, Kazhdan's property $T$, strong ergodicity and invariant means for ergodic group-actions
}

\author{
KLAUS SCHMIDT $\dagger$ \\ From the Mathematics Institute, University of Warwick, England
}

(Received 15 November 1980)

\begin{abstract}
This paper discusses the relations between the following properties of finite measure preserving ergodic actions of a countable group $G$ : strong ergodicity (i.e. the non-existence of almost invariant sets), uniqueness of $G$-invariant means on the measure space carrying the group action, and certain cohomological properties. Using these properties one can characterize all actions of amenable groups and of groups with Kazhdan's property $T$. For groups which fall in between these two definitions these notions lead to some interesting examples.
\end{abstract}

\section{Introduction}

The aim of this paper is to clarify the relation between a number of concepts and recent results concerning finite measure preserving ergodic actions of a countable group on a probability space $(X, \mathscr{S}, \mu)$. By definition, an ergodic action of a countable group $G$ will not have any non-trivial invariant sets. It may happen, however, that $G$ admits sets which are 'almost' invariant (for a precise definition we refer to $\S 2$ ). Following [2] we call a finite measure preserving ergodic action of $G$ strongly ergodic if it admits no almost invariant sets. From Rokhlin's lemma it is clear that single ergodic transformations can never be strongly ergodic [15], and a recent result by Connes, Feldman and Weiss implies that no measure preserving action of an amenable group $G$ can ever be strongly ergodic. The situation is quite different for groups satisfying Kazhdan's condition $T$ (like $\operatorname{SL}(n, \mathbb{Z}), n \geq 3$, for example): they can be characterized as precisely those groups for which every finite measure preserving ergodic action is strongly ergodic [2]. In three recent papers [4], [7], [12], another concept has been introduced which is fairly closely related to strong ergodicity: the uniqueness of $G$-invariant means on $L^{\infty}(X, \mathscr{S}, \mu)$ (this means that integration is the unique $G$-invariant mean on $\left.L^{\infty}(X, \mathscr{P}, \mu)\right)$. Again one can characterize amenable groups as those groups for which no finite measure preserving action admits a unique $G$-invariant mean, and groups with property $T$ as those for which every finite measure preserving ergodic action has a unique invariant mean. Amenability and property $T$ are, however, not complementary notions, and there exist groups which fall between these two definitions, like $\operatorname{SL}(2, \mathbb{Z})$, for example, or like any free group on more than one generator of infinite order. For these groups

$\dagger$ Address for correspondence: Dr Klaus Schmidt, Mathematics Institute, University of Warwick, Coventry CV4 7AL, England. 
there exist finite measure preserving ergodic actions on $(X, \mathscr{Y}, \mu)$ which are strongly ergodic and others which are not, and there exist actions with and without unique $G$-invariant means. Furthermore, one can have strongly ergodic actions without a unique invariant mean, but not the other way round.

$\S 3$ studies the relations between these various notions and certain properties of the first cohomology groups of $G$ on $(X, \mathscr{S}, \mu)$. Again one can characterize both amenability and property $T$ by cohomological properties, and can find some interesting examples and conclusions for groups falling into the gap between amenability and property $T$. $\$ 4$ deals with the characterization of property $T$ in terms of cohomology with values in an amenable group $A$. This section also contains a general necessary and sufficient condition for a cocycle of a countable group $G$ to be cohomologous to a cocycle with values in a compact subgroup.

While a final version of this article was being prepared the author received a preprint by $\mathrm{R}$. Zimmer [17] containing some of the results of this paper: in particular [17] contains the implications $(4) \Rightarrow(1),(4) \Rightarrow(2)$ and $(4) \Rightarrow(3)$ of theorem 3.4 , and proposition 4.1 of this paper.

\section{Strong ergodicity and invariant means}

Let $G$ be a countable group and let $B$ be a complex separable Banach space. A representation $V: g \rightarrow V_{g}$ of $G$ by linear isometries of $B$ is said to contain the trivial representation weakly if there exists a sequence $\left(v_{n}\right)$ in $B$ with $\left\|v_{n}\right\|=1$ for every $n$ and with $\lim _{n}\left\|v_{n}-V_{g} v_{n}\right\|=0$ for every $g \in G$. In the special case where there exists a non-zero vector $v \in B$ with $V_{g} v=v$ for every $g \in G$, we say that $V$ contains the trivial representation. Let now $(X, \mathscr{P}, \mu)$ be a fixed standard non-atomic probability space and let $(g, x) \rightarrow g x$ be a measure preserving ergodic action of $G$ on $(X, \mathscr{P}, \mu)$. For every $p$ with $1 \leq p<\infty$ we define a representation $U^{(p)}$ of $G$ by isometries of $L^{p}(X, \mathscr{P}, \mu)$ by setting

$$
\left(U_{g}^{(p)} f\right)(x)=f\left(g^{-1} x\right), \quad x \in X,
$$

for every $g \in G, f \in L^{P}(X, \mathscr{S}, \mu) . L_{0}^{P}(X, \mathscr{P}, \mu)$ will denote the space

$$
\left\{f \in L^{p}(X, \mathscr{S}, \mu) ; \int f d \mu=0\right\}
$$

and $V^{(p)}$ the restriction of $U^{(p)}$ to $L_{0}^{p}(X, \mathscr{S}, \mu)$. The ergodicity of $G$ implies that $V^{(p)}$ does not contain the trivial representation. It may happen, however, that $V^{(p)}$ contains the trivial representation weakly.

A sequence $\left(C_{n}, n \geq 1\right)$ in $\mathscr{S}$ is called an $I$-sequence ( $I$ stands for invariance) if $\mu\left(C_{n}\right)>0$ for every $n, \lim _{n} \mu\left(C_{n}\right)=0$, and $\lim _{n} \mu\left(C_{n} \Delta g C_{n}\right) / \mu\left(C_{n}\right)=0$ for every $g \in G$ (cf. [4]). For our next definition we assume that $\Gamma$ is a (not necessarily countable) group and $(\gamma, x) \rightarrow \gamma x$ an ergodic, measure preserving action of $\Gamma$ on $(X, \mathscr{P}, \mu)$. A $\Gamma$-invariant mean is a linear functional $M: L^{\infty}(X, \mathscr{S}, \mu) \rightarrow \mathbb{C}$ with $M(1)=1, M(f) \geq 0$ for $f \geq 0$, and with $M(f \cdot \gamma)=M(f)$ for every $f \in L^{\infty}(X, \mathscr{S}, \mu)$ and $\gamma \in \Gamma$. We quote the following result from [12]. 
(2.1) LEMMA. The following two conditions are equivalent.

(1) $G$ admits an $I$-sequence in $\mathscr{S}$.

(2) $G$ has more than one invariant mean on $L^{\infty}(X, \mathscr{S}, \mu)$.

Although some of the results in this section will have obvious analogues in the case of uncountable groups, we shall deal almost exclusively with countable groups in order to keep notation simple. If $G$ is a countable group and $(g, x) \rightarrow g x$ a measure preserving ergodic action of $G$ on $(X, \mathscr{S}, \mu)$, we call a sequence $\left(C_{n}\right) \subset \mathscr{S}$ asymptotically invariant under $G$ if $\lim _{n} \mu\left(C_{n} \triangle g C_{n}\right)=0$ for every $g \in G$ (cf. [15]). An asymptotically invariant (a.i.) sequence $\left(C_{n}\right)$ is said to be trivial if

$$
\lim _{n} \mu\left(C_{n}\right) \cdot\left(1-\mu\left(C_{n}\right)\right)=0,
$$

and the action of $G$ is called strongly ergodic if every a.i. sequence $\left(C_{n}\right)$ in $\mathscr{S}$ is trivial. If the action of $G$ is not strongly ergodic, it admits many a.i. sequences. In fact, one can find, for every $\varepsilon$ with $0<\varepsilon<1$, and an a.i. sequence $\left(C_{n}\right) \subset \mathscr{S}$ with $\mu\left(C_{n}\right)=\varepsilon$ for every $n$. We mention in passing that strong ergodicity is an orbit-equivalence invariant (see [15] and theorem 2.6 of this paper). From the abundance of a.i. sequences for actions which are not strongly ergodic and from lemma 2.1 one concludes immediately:

(2.2) Proposition. If the action of $G$ on $(X, \mathscr{S}, \mu)$ is not strongly ergodic then $G$ has more than one invariant mean on $L^{\infty}(X, \mathscr{S}, \mu)$.

(2.3) Proposition. Let $G$ be a countable group and let $(g, x) \rightarrow g x$ be a measure preserving ergodic action of $G$ on $(X, \mathscr{S}, \mu)$. The following conditions are equivalent.

(1) There exists a $p$ with $1 \leq p<\infty$ such that $V^{(p)}$ contains the trivial representation weakly.

(2) For every $p$ with $1 \leq p<\infty, V^{(p)}$ contains the trivial representation weakly.

(3) $G$ admits more than one invariant mean on $L^{\infty}(X, \mathscr{P}, \mu)$.

Proof. The implication $(2) \Rightarrow(1)$ is trivial. To prove $(3) \Rightarrow(2)$, assume that the action of $G$ admits more than one invariant mean. By lemma 2.1 and the countability of $G$ we can find an $I$-sequence $\left(C_{n}\right)$ in $\mathscr{S}$. Put $h_{n}(x)=\chi_{C_{n}}(x)-\mu\left(C_{n}\right)$, where $\chi_{C_{n}}$ is the indicator function of $C_{n}$. We fix $p$ and put $f_{n}=h_{n} /\left\|h_{n}\right\|_{p}, n \geq 1$, where $\|\cdot\|_{p}$ denotes the $p$-norm. One checks easily that $\lim \left\|f_{n}-V_{g}^{(p)} f_{n}\right\|_{p}=0$ for every $g \in G$, so that (2) is satisfied. The remaining implication ${ }^{n}(1) \Rightarrow(3)$ is slightly more involved, although the proof is quite standard. Assume that (1) is satisfied, and choose a sequence of real-valued functions $\left(f_{n}\right)$ in $L_{0}^{p}(X, \mathscr{S}, \mu)$ with $\left\|f_{n}\right\|_{p}=1$ for every $n$ and with

$$
\lim _{n}\left\|f_{n}-V_{\mathrm{g}}^{(p)} f_{n}\right\|_{p}=0
$$

for every $g \in G$. We define a sequence $\left(\sigma_{n}\right)$ of probability measures on $\mathbb{R}$ by setting $\sigma_{n}(D)=\mu\left(\left\{x: f_{n}(x) \in D\right\}\right), D \subset \mathbb{R}$, and observe that this sequence $\left(\sigma_{n}\right)$ is uniformly tight [10, theorem 6.7]. Without loss of generality we may therefore assume that $\left(\sigma_{n}\right)$ converges weakly to a probability measure $\sigma$ on $\mathbb{R}$. If $\sigma$ is not concentrated in a single point, we can find an $\alpha \in \mathbb{R}$ with $\sigma(\{\alpha\})=0$ and with $0<\sigma((-\infty, \alpha))<1$. Since $\alpha$ is a continuity point of $\sigma$, we have $0<\lim _{n} \sigma_{n}((-\infty, \alpha))=$ 
$\sigma((-\infty, \alpha))<1$, and, putting $C_{n}=\left\{x: f_{n}(x)<\alpha\right\}$, we obtain $\operatorname{limin}_{n} \mu\left(C_{n} \Delta g C_{n}\right)=0$ for every $g \in G$. The action of $G$ on $(X, \mathscr{S}, \mu)$ is thus not strongly ergodic, and proposition 2.2 implies (3). To complete the proof we have to deal with the case where $\sigma$ is concentrated in a single point $\alpha_{0}$. Since

$$
\begin{aligned}
& \int \|\left. f_{n}(x)\right|^{p}-\left|V_{8}^{(p)} f_{n}(x)\right|^{p} \mid d \mu(x) \\
& \quad=\int \|\left. f_{n}(x)\right|^{p}-\left|f_{n}\left(g^{-1} x\right)\right|^{p} \mid d \mu(x) \\
& \quad \leq \int\left\|f_{n}(x)|-| f_{n}\left(g^{-1} x\right)\right\| \cdot p \cdot\left(\left|f_{n}(x)\right|^{p-1}+\left|f_{n}\left(g^{-1} x\right)\right|^{p-1}\right) d \mu(x) \\
& \quad \leq 2 p \cdot\left\|f_{n}-f_{n} \cdot g^{-1}\right\|_{p} \cdot\left\|f_{n}\right\|_{p}^{p-1},
\end{aligned}
$$

we obtain, for $h_{n}=\left|f_{n}\right|^{p}$,

$$
\int h_{n} d \mu=1, n \geq 1
$$

and

$$
\lim _{n}\left\|h_{n}-h_{n} \cdot g^{-1}\right\|_{1}=0
$$

for every $g \in G$. Let now, for every $n \geq 1$,

$$
h_{n}^{*}(x)= \begin{cases}h_{n}(x) & \text { if } h_{n}(x) \geq\left|\alpha_{0}\right|^{p}+1, \\ \left|\alpha_{0}\right|^{p} & \text { otherwise. }\end{cases}
$$

Since $\sigma$ is concentrated in $\alpha_{0}$, and since $\left\|f_{n}\right\|_{p}=1$ for all $n$, the functions $h_{n}^{*}$ will satisfy

$$
\begin{gathered}
\lim _{n} \int h_{n}^{*} d \mu=1, \\
\lim _{n} \operatorname{ess} \sup h_{n}^{*}=\infty,
\end{gathered}
$$

and

$$
\lim _{n}\left\|h_{n}^{*}-h_{n}^{*} \cdot g^{-1}\right\|_{1}=0
$$

for every $g \in G$. Using a technique due to I. Namioka we set, for every $s \in \mathbb{R}$, $x \in X, g \in G$,

$$
F_{n}(g, s, x)= \begin{cases}1 & \text { if } s \leq h_{n}^{*}\left(g^{-1} x\right) \\ 0 & \text { otherwise }\end{cases}
$$

and obtain

$$
\begin{aligned}
\left\|h_{n}^{*}-h_{n}^{*} \cdot g^{-1}\right\|_{1} & =\iint_{0}^{\infty}\left|F_{n}(e, s, x)-F_{n}(g, s, x)\right| d s d \mu(x) \\
& =\int_{0}^{\infty} \int\left|F_{n}(e, s, x)-F_{n}(g, s, x)\right| d \mu(x) d s,
\end{aligned}
$$

where $e$ denotes the identity element in $G$ (this adaptation of I. Namioka's method is due to A. Connes). Furthermore,

$$
\left\|h_{n}^{*}\right\|_{1}=\iint_{0}^{\infty} F_{n}(e, s, x) d s d \mu(x)=\int_{0}^{\infty} \int F_{n}(e, s, x) d \mu(x) d s
$$


Let now $\left(G_{k}\right)$ be an increasing sequence of finite subsets of $G$ with $G=\bigcup G_{k}$ and choose, for every $k \geq 1$, an integer $n_{k} \geq 1$ with

$$
\sum_{g \in G_{k}}\left\|h_{n_{k}}^{*}-h_{n_{k}}^{*} \cdot g^{-1}\right\|_{1}<\frac{1}{k \cdot\left\|h_{n_{k}}^{*}\right\|_{1}}
$$

The inequality

$$
\sum_{g \in G_{k}} \int_{0}^{\infty} \int\left|F_{n_{k}}(e, s, x)-F_{n_{k}}(g, s, x)\right| d \mu(x) d s<\frac{1}{k} \cdot \int_{0}^{\infty} \int F_{n_{k}}(e, s, x) d \mu(x) d s,
$$

which is a consequence of (2.1)-(2.3), implies the existence of a real number $s(k)>\left|\alpha_{0}\right|^{p}$ with

$$
\begin{aligned}
0 & \leq \int \sum_{g \in G_{k}}\left|F_{n_{k}}(e, s(k), x)-F_{n_{k}}(g, s(k), x)\right| d \mu(x) \\
& <\frac{1}{k} \cdot \int F_{n_{k}}(e, s(k), x) d \mu(x)
\end{aligned}
$$

Put

$$
C_{k}=\left\{x: h_{n_{k}}^{*}(x) \geq s(k)\right\} .
$$

Our assumption on $\left(\sigma_{n}\right)$ implies that $\lim _{k} \mu\left(C_{k}\right)=0$, and (2.4) yields

$$
\sum_{g \in G_{k}} \mu\left(C_{k} \triangle g C_{k}\right)<\frac{1}{k} \cdot \mu\left(C_{k}\right)
$$

We have proved the existence of an $I$-sequence for $G$ in $\mathscr{P}$, and (3) is now implied by lemma 2.1. The proof is complete.

Propositions 2.2 and 2.3 now allow us to turn to the main results of this section.

(2.4) ThEOREM. Let $G$ be a countable group and let $(X, \mathscr{S}, \mu)$ be a standard non-atomic probability space. The following conditions are equivalent.

(1) No ergodic measure preserving action of $G$ on $(X, \mathscr{S}, \mu)$ is strongly ergodic.

(2) No ergodic measure preserving action of $G$ on $(X, \mathscr{S}, \mu)$ has a unique $G$ invariant mean on $L^{\infty}(X, \mathscr{S}, \mu)$.

(3) $G$ is amenable.

Proof. (1) $\Rightarrow(2)$ follows from proposition 2.2. The implication $(2) \Rightarrow(3)$ has been proved in [7]: assume that $G$ is not amenable and let $K$ be a compact metric abelian group with Haar measure $\lambda$ and dual group $\hat{K}$. Put $X^{\prime}=K^{G}$ (the space of all functions $x: G \rightarrow K$ ) and $\mu^{\prime}=\lambda^{G}$ (the product measure). $X^{\prime}$ is a compact metric abelian group with Haar measure $\mu^{\prime}$ under the product topology and pointwise addition as composition. For $g \in G$ and $x \in X^{\prime}$, let $g \cdot x(h)=x\left(g^{-1} h\right)$. This 'Bernoulli'-action of $G$ on $\left(X^{\prime}, \mathscr{S}^{\prime}, \mu^{\prime}\right)$, where $\mathscr{S}^{\prime}$ is the product Borel field, is ergodic and measure preserving, and the adjoint action of $G$ on the dual group $\hat{X}^{\prime}$ of $X^{\prime}$ is free on the complement of $\{1\}$ ( 1 is the identity element in $\left.\hat{X}^{\prime}\right)$. From this and from the non-amenability of $G$ it is easy to see that this action of $G$ on $\left(X^{\prime}, \mathscr{S}^{\prime}, \mu^{\prime}\right)$ has the property that the representation $V^{(2)}$ of $G$ on $L_{0}^{2}\left(X^{\prime}, \mathscr{S}_{,}^{\prime}, \mu^{\prime}\right)$, given by

$$
\left(V_{\mathrm{g}}^{(2)} f\right)(x)=f\left(g^{-1} \cdot x\right), \quad x \in X^{\prime}, \quad f \in L_{0}^{(2)}\left(X^{\prime}, \mathscr{S}^{\prime}, \mu^{\prime}\right), \quad g \in G,
$$


does not contain the trivial representation weakly (cf. [6, p. 61]). Proposition 2.3 now implies that this action of $G$ has a unique invariant mean on $L^{\infty}\left(X^{\prime}, \mathscr{S}^{\prime}, \mu^{\prime}\right)$, and we conclude that (2) implies ( 3 ). The final implication ( 3$) \Rightarrow(1)$ follows from the fact that every (measure preserving) action of $G$ on $(X, \mathscr{S}, \mu)$ is approximately finite (cf. [3]) and hence not strongly ergodic (cf. [15]). An application of proposition 2.2 completes the proof of the theorem.

For our next result we recall that a countable group $G$ is said to satisfy Kazhdan's condition $T$ if the trivial representation is an isolated point in the dual space $\hat{G}$ of $G$ or, equivalently, if, for every unitary representation $W$ of $G$ on a complex separable Hilbert space, $W$ contains the trivial representation weakly if and only if $W$ contains the trivial representation.

(2.5) Theorem. Let $G$ be a countable group and let $(X, \mathscr{S}, \mu)$ be a standard non-atomic probability space. The following conditions are equivalent.

(1) Every ergodic measure preserving action of $G$ on $(X, \mathscr{S}, \mu)$ is strongly ergodic.

(2) Every ergodic measure preserving action of $G$ on $(X, \mathscr{Y}, \mu)$ has a unique $G$-invariant mean on $L^{\infty}(X, \mathscr{P}, \mu)$.

(3) $G$ has property $T$ of Kazhdan.

Proof. The equivalence of (1) and (3) is the subject of [2]. (3) $\Rightarrow(2)$ by proposition 2.3 , and $(2) \Rightarrow(1)$ follows from proposition 2.2 .

Although the uniqueness of $G$-invariant means distinguishes amenable groups from groups with property $T$ as long as these groups are countable, the situation is quite different for full groups. Recall that the full group $[G]$ of an action of $G$ on $(X, \mathscr{S}, \mu)$ consists of all automorphisms $S$ of $(X, \mathscr{S}, \mu)$ with $\left\{S^{n} x: n \in \mathbb{Z}\right\} \subset\{g x: g \in G\}$ for $\mu$-a.e. $x \in X$. I am grateful to $H$. A. Dye for pointing out this fact to me and for allowing me to include the following proof.

(2.6) THEOREM. Let $G$ be a countable group and let $(g, x) \rightarrow g x$ be an ergodic measure preserving action of $G$ on a standard non-atomic probability space $(X, \mathscr{S}, \mu)$. Then $[G]$ has a unique invariant mean on $L^{\infty}(X, \mathscr{S}, \mu)$.

Proof. Let $M$ be a $[G]$-invariant mean on $L^{\infty}(X, \mathscr{S}, \mu)$ with $M(f) \neq \int f d \mu$ for some $f \in L^{\infty}(X, \mathscr{S}, \mu)$. Without loss in generality we may assume that $M$ is singular, i.e. that there exists a set $B \in \mathscr{S}$ with $\mu(B)>0$, but $M\left(\chi_{B}\right)=0$. By decreasing $B$ we may assume that $\mu(B)=1 / n$ for some $n>1$, and we choose elements $S_{1}, \ldots, S_{n}$ in $[G]$ such that $S_{1} B=B, S_{2} B, \ldots, S_{n} B$ forms a partition of $X$. We obtain $1=M(1)=$ $\sum_{k=1}^{n} M\left(\chi s_{k} B\right)=0$, and this contradiction proves our assertion.

Although theorems 2.4 and 2.5 might suggest the equivalence of strong ergodicity and the uniqueness of a $G$-invariant mean, the actual situation is more complicated and we conclude this section with an example which shows that there exists a measure preserving ergodic action of a countable group $G$ on a standard non-atomic probability space $(X, \mathscr{P}, \mu)$ which is strongly ergodic, but which has more than one $G$-invariant mean on $L^{\infty}(X, \mathscr{S}, \mu)$. 
(2.7) Example. In the following we shall construct a finite measure preserving ergodic action of the free group $G$ on three generators $a, b$, $c$, which is strongly ergodic, but which has more than one invariant mean. Let $G_{2}$ be the free subgroup of $G$ generated by $a$ and $b$, and let $(Y, \mathscr{T}, \nu)$ be the 2-torus with its usual Borel field and Lebesgue measure. The two matrices $\left(\begin{array}{ll}1 & 1 \\ 0 & 1\end{array}\right)$ and $\left(\begin{array}{ll}1 & 0 \\ 1 & 1\end{array}\right)$ act as linear automorphisms of $(Y, \mathscr{T}, \nu)$, and one can either prove directly or as a corollary to the results in [15] that the action of $G_{2}$ defined by letting $a$ act as $\left(\begin{array}{ll}1 & 1 \\ 0 & 1\end{array}\right)$ and $b$ as $\left(\begin{array}{ll}1 & 0 \\ 1 & 1\end{array}\right)$ on $(Y, \mathscr{T}, \nu)$ is ergodic, measure preserving and strongly ergodic (in [15] we prove that the action of all of $\operatorname{SL}(2, \mathbb{Z})$ on $(Y, \mathcal{T}, \nu)$ is strongly ergodic). Let now $X=Y \times \mathbb{N}$, where $\mathbb{N}=\{1,2, \ldots\}$, and let $\mathscr{S}$ be the product Borel field of $X . \mu$ will denote the probability measure on $(X, \mathscr{S})$ given by

$$
\mu(B)=\frac{6}{\pi^{2}} \cdot \sum_{n=1}^{\infty} n^{-2} \cdot \nu(\{y \in Y:(y, n) \in B\})
$$

for every $B \in \mathscr{S}$. For every $g \in G_{2},(y, n) \in X$, put $g(y, n)=(g y, n)$, where $(g, y) \rightarrow g y$ is the earlier described action on $G_{2}$ of $(Y, \mathscr{T}, \nu)$. The action of $G_{2}$ on $(X, \mathscr{S}, \mu)$ is again measure preserving, but not ergodic. We shall now describe the action of the third generator $c$ of $G$ on $(X, \mathscr{S}, \mu)$. We choose a measure preserving automorphism $S$ of $(X, \mathscr{P}, \mu)$ such that

$$
S\left(Y_{n}\right) \subset Y_{n-1}
$$

for every $n \geq 2$, where $Y_{n}=\{(y, n): y \in Y\}$, and represent $c$ on $(X, \mathscr{S}, \mu)$ by $S$. Since the action of $G_{2}$ on $(Y, \mathscr{T}, \nu)$ is strongly ergodic, one can check quite easily that the action of $G$ on $(X, \mathscr{Y}, \mu)$ is again strongly ergodic. On the other hand, if we set $C_{n}=\bigcup_{m \geq n} Y_{n}, n \geq 1$, we see that $a C_{n}=b C_{n}=C_{n}$ for every $n \geq 1$, and that $\lim _{n} \mu\left(C_{n} \triangle c C_{n}\right) / \mu\left(C_{n}\right)=0$. An elementary computation now implies that $\lim _{n}^{n} \mu\left(C_{n} \Delta g C_{n}\right) / \mu\left(C_{n}\right)=0$ for every $g \in G$. Using lemma 2.1 we conclude that $G$ has more than one invariant mean on $L^{\infty}(X, \mathscr{S}, \mu)$.

\section{Cohomology and property $T$}

Let $G$ be a countable group, $(X, \mathscr{S}, \mu)$ a standard non-atomic probability space, $(g, x) \rightarrow g x$ a non-singular ergodic action of $G$ on $(X, \mathscr{S}, \mu)$ and $A$ a locally compact second countable group. $U(X, \mu, A)$ stands for the group of $\mu$-equivalence classes of Borel maps $\phi: X \rightarrow A$, furnished with the topology of convergence in measure and pointwise multiplication as composition. We note that $U(X, \mu, A)$ is a polish group and carries a natural $G$-action given by $(g, \phi) \rightarrow \phi \cdot g^{-1}$. A map $a: G \rightarrow U(X, \mu, A)$ is called a (1-)cocycle for $G$ if

$$
a\left(g_{1}, g_{2} x\right) \cdot a\left(g_{2}, x\right)=a\left(g_{1} g_{2}, x\right)
$$

for every $g_{1}, g_{2} \in G$ and for $\mu$-a.e. $x \in X$ (we are writing $a(g, x)$ instead of $a(g)(x)$ ). 
A cocycle $a: G \rightarrow U(X, \mu, A)$ is said to be a coboundary if there exists a Borel map $b \in U(X, \mu, A)$ with

$$
a(g, x)=b(g x)^{-1} b(x)
$$

for every $g \in G$ and $\mu$-a.e. $x \in X$. Finally, we call two cocycles $a_{i}, i=1,2$, cohomologous if there exists a $b \in U(X, \mu, A)$ with

$$
a_{1}(g, x)=b(g x)^{-1} a_{2}(g, x) b(x)
$$

for every $g \in G$ and $\mu$-a.e. $x \in X . Z^{1}(G, U(X, \mu, A))$ will denote the set of cocycles for $G$ and $B^{1}(G, U(X, \mu, A))$ the subset of coboundaries. $Z^{1}(G, U(X, \mu, A))$ is a polish space in the topology of pointwise convergence on $G$.

If $A$ is abelian, $Z^{1}(G, U(X, \mu, A))$ is an abelian group and $B^{1}(G, U(X, \mu, A))$ a subgroup. $H^{1}(G, U(X, \mu, A))$ will then denote the quotient group $Z^{1}(G, U(X, \mu, A)) / B^{1}(G, U(X, \mu, A))$. For the general background of these definitions we refer to [5], [13] and [16]. For $A=\mathbb{C}$ and for a measure preserving action of $G$ on $(X, \mathscr{S}, \mu)$ we define further cohomology groups $H^{1}\left(G, L^{p}(X, \mathscr{P}, \mu)\right)=$ $Z^{1}\left(G, L^{p}(X, \mathscr{S}, \mu)\right) / B^{1}\left(G, L^{p}(X, \mathscr{S}, \mu)\right)$ by replacing $U(X, \mu, A)$ everywhere by $L^{p}(X, S, \mu)$ for some $p$ with $1 \leq p<\infty$. Again $Z^{1}\left(G, L^{p}(X, \mathscr{S}, \mu)\right)$ is a polish group under the topology of pointwise convergence on $G$.

(3.1) Theorem. Let $G$ be a countable group, $(X, \mathscr{S}, \mu)$ a standard non-atomic probability space, and $(g, x) \rightarrow g x$ a measure preserving ergodic action of $G$ on $(X, \mathscr{P}, \mu)$. The following conditions are equivalent.

(1) There exists a $p$ with $1 \leq p<\infty$ such that $B^{1}\left(G, L^{p}(X, \mathscr{S}, \mu)\right)$ is a closed subgroup of $Z^{1}\left(G, L^{p}(X, \mathscr{S}, \mu)\right)$.

(2) The representation $V^{(p)}$ of $G$ on $L_{0}^{p}(X, \mathscr{S}, \mu)$ does not contain the trivial representation weakly, for some $p$ with $1 \leq p<\infty$.

(3) (1) and (2) are true for every $p$ with $1 \leq p<\infty$.

(4) $G$ has a unique invariant mean on $L^{\infty}(X, \mathscr{S}, \mu)$.

Proof. This is an immediate consequence of proposition 2.3.

(3.2) TheOREM. Let $G$ be a countable group, $(X, \mathscr{S}, \mu)$ a standard non-atomic probability space, and $(g, x) \rightarrow g x$ a measure preserving ergodic action of $G$ on $(X, \mathscr{S}, \mu)$. The following conditions are equivalent.

(1) There exists a locally compact second countable abelian group $A \neq\{0\}$ such that $B^{1}(G, U(X, \mu, A))$ is a closed subgroup of $Z^{1}(G, U(X, \mu, A))$.

(2) For every locally compact second countable abelian group $A, B^{1}(G, U(X, \mu, A))$ is a closed subgroup of $Z^{1}(G, U(X, \mu, A))$.

(3) The action of $G$ on $(X, \mathscr{S}, \mu)$ is strongly ergodic.

Proof. This is theorem 2.4 in [15].

For the following lemma we assume again that $G$ is a countable group and $(g, x) \rightarrow g x$ a measure preserving ergodic action of $G$ on $(X, \mathscr{S}, \mu)$. For a fixed $c \in$ $Z^{1}(G, U(X, \mu, \mathbb{T}))\left(\mathbb{T}\right.$ is the circle group), consider the representation $U^{c}$ of $G$ on 
$L^{2}(X, \mathscr{S}, \mu)$ given by

$$
\left(U_{g}^{c} f\right)(x)=c\left(g^{-1}, x\right) f\left(g^{-1} x\right), \quad x \in X,
$$

for every $g \in G$ and $f \in L^{2}(X, \mathscr{S}, \mu)$.

(3.3) LEMMA. $U^{c}$ contains the trivial representation if and only if $c$ is a coboundary. For every $c \in \overline{B^{1}(G, U(X, \mu, \mathbb{T}))}$ (the bar denotes closure in $Z^{1}(G, U(X, \mu, \pi))$ ), $U^{c}$ contains the trivial representation weakly.

Proof. From (3.4) it is obvious that any function $f_{0} \in L^{2}(X, \mathscr{S}, \mu)$ which is invariant under $U^{c}$ must have constant modulus and cobound $c$. The second assertion is equally obvious: if $c(g, x)=\lim _{n} b_{n}(g x)^{-1} b_{n}(x)$ in $\mu$-measure, where $b_{n} \in U(X, \mu, \mathbb{V})$, then $\left(b_{n}\right)$, considered as a sequence in $L^{2}(X, \mathscr{S}, \mu)$, will be asymptotically invariant under $U^{c}$. The lemma is proved.

(3.4) Theorem. Let $G$ be a countable group and $(X, \mathscr{S}, \mu)$ a standard non-atomic probability space. The following conditions are equivalent.

(1) For every locally compact second countable abelian group $A$, every measure preserving ergodic action $(g, x) \rightarrow g x$ of $G$ on $(X, \mathscr{S}, \mu)$, and for every $a \in Z^{1}(G, U(X, \mu, A))$ there exists a compact subgroup $K$ of $A$ and a cocycle $a^{\prime}$ cohomologous to a and taking values in $K$.

(2) For every measure preserving ergodic action of $G$ on $(X, \mathscr{S}, \mu)$, $H^{1}(G, U(X, \mu, \mathbb{Z}))=0$.

(3) For every measure preserving ergodic action of $G$ on $(X, \mathscr{S}, \mu)$, $H^{1}(G, U(X, \mu, T))$ is countable.

(4) $G$ has Kazhdan's property $T$.

Proof. (1) $\Rightarrow(2)$ is obvious. To prove (2) $\Rightarrow(4)$ note that (2) implies in particular that $B^{1}(G, U(X, \mu, \mathbb{Z}))$ is a closed subgroup of $Z^{1}(G, U(X, \mu, \mathbb{Z}))$. Theorem 3.2 and theorem 2.5 combine to give (4). Assume now that (4) is satisfied. Kazhdan's property $T$ implies that there exists a finite set $G_{0}$ in $G$ and an $\varepsilon>0$ with the following property: if $W$ is any unitary representation of $G$ on a complex separable Hilbert space $H$, and if there exists an element $f \in H$ with $\|f\|=1$ and $\max _{\mathbf{g} \in G_{0}}\left\|W_{\mathrm{g}} f-f\right\|<\varepsilon$ then $W$ contains the trivial representation. To apply this condition. we fix a measure preserving ergodic action of $G$ on $(X, \mathscr{P}, \mu)$ and a $c_{0} \in$ $B^{1}(G, U(X, \mu, \mathbb{T}))$. By lemma $3.3, U^{c_{0}}$ has a fixed vector $f_{0} \in L^{2}(X, \mathscr{S}, \mu)$ with unit norm. For every $c \in Z^{1}(G, U(X, \mu, \pi))$ with $\left.\int \mid c\left(g^{-1}, x\right)-c_{0}\left(g^{-1}, x\right)\right) \mid d \mu(x)<\delta$ for every $g \in G_{0}$, where $\delta$ is sufficiently small, we obtain $\left\|U_{g}^{c} f_{0}-f_{0}\right\|<\varepsilon$ for every $g \in G_{0}$. $U^{c}$ will thus contain the trivial representation, so that $c$ is a coboundary. We have proved that $B^{1}(G, U(X, \mu, \mathbb{T}))$ is an open subgroup of $Z^{1}(G, U(X, \mu, \mathbb{T}))$. Hence $H^{1}$ is discrete, and countable on account of its separability. Having proved that (4) implies (3) we now turn to the remaining implication (3) $\Rightarrow(1)$. Let $A$ be a locally compact second countable abelian group, $\hat{A}$ its dual group, and let $a \in$ $Z^{1}(G, U(X, \mu, A))$. For every $\chi \in \hat{A}$, let $c_{\chi}$ denote the cocycle $\chi \cdot a$ in 
$Z^{1}(G, U(X, \mu, \pi))$. The map $\chi \rightarrow c_{\chi}$ is continuous from $\hat{A}$ into $Z^{1}(G, U(X, \mu, \pi))$ and hence defines a continuous map $X \rightarrow\left[c_{\chi}\right]$ from $\hat{A}$ into $H^{1}(G, U(X, \mu, \mathbb{T})$ ) (note that $B^{1}$ is closed as a consequence of (3)). Since $H^{1}$ is countable, [ $\left.c_{X}\right]$ must be trivial for all $\chi$ in a neighbourhood $N(1)$ of 1 in $\hat{A}$. Let $\hat{A}_{0}$ be the open subgroup generated by $N(1)$. Then $\hat{A}_{0}=A / K$ for some compact subgroup $K$ of $A$. The cocycle $\left.a^{*}: G \rightarrow U(X, \mu, A / K)\right)$, given by $a^{*}(g, x)=a(g, x)+K$, is a coboundary by [13]. A standard argument now shows that $a$ is cohomologous to a cocycle $a^{\prime}$ with $a^{\prime}(g, x) \in$ $K$ for every $g \in G$ and a.e. $x \in X$. The proof is complete.

(3.5) Remark. The equivalence of (3) and (4) is a special case of a result in [1], and the implication $(4) \Rightarrow(1)$ has also been noticed by $\mathrm{H}$. Furstenberg. It is interesting to compare the statement of theorem 3.4 with the following assertion which is a consequence of [11] and of theorem 2.4: acountable group $G$ is amenable if and only if, for every measure preserving ergodic action of $G$ on $(X, \mathscr{S}, \mu)$, and for every locally compact second countable abelian group $A, B^{1}(G, U(X, \mu, A)) \neq$ $\overline{B^{1}(G, U(X, \mu, A))}=Z^{1}(G, U(X, \mu, A))$.

Let $G$ and $\Gamma$ be two countable groups with measure preserving ergodic actions $(g, x) \rightarrow g x$ and $(\gamma, x) \rightarrow \gamma x$ on $(X, \mathscr{S}, \mu)$. The two actions are called orbit equivalent if there exists a (necessarily measure preserving) automorphism $V$ of $(X, \mathscr{S}, \mu)$ with $V[G] V^{-1}=[\Gamma]$. If $G=\Gamma$ one can ask whether two different actions of $G$ are orbit equivalent. The results in [3] imply that any two measure preserving ergodic actions of a countable amenable group $G$ are orbit equivalent, since they must both be approximately finite. Theorem 2.4 and theorem 2.5 have the following corollary.

(3.6) COROllaRy. Let $G$ be a countable group which is neither amenable nor satisfying property $T$. Then $G$ has two measure preserving ergodic actions on $(X, \mathscr{S}, \mu)$ (namely one which is strongly ergodic and one which is not).

(3.7) Example. Let $G=\operatorname{SL}(2, \mathbb{Z})$, acting as the group of linear automorphisms on the 2-torus $(Y, \mathscr{T}, \nu)$ (cf. example 2.7). This action is strongly ergodic (cf. [15]). Consider now the cocycle $a \in Z^{1}(G, U(Y, \nu, \mathbb{Z}))$ satisfying

$$
a\left(\left(\begin{array}{rr}
-1 & 0 \\
0 & -1
\end{array}\right), y\right)=a\left(\left(\begin{array}{rr}
0 & -1 \\
1 & 1
\end{array}\right), y\right)=0, \quad y \in Y,
$$

and

$$
a\left(\left(\begin{array}{rr}
0 & -1 \\
1 & 0
\end{array}\right), y\right)=\left\{\begin{aligned}
-1 & \text { for } y=(s, t) \text { with } 0 \leq s, t<\frac{1}{2} \text { and } \frac{1}{2} \leq s, t<1 \\
1 & \text { otherwise. }
\end{aligned}\right.
$$

As shown in [15], $a$ is a well defined cocycle for $\operatorname{SL}(2, \mathbb{Z})$, and the corresponding infinite measure preserving skew product action of $G$ on $Y \times \mathbb{Z}$, given by $g(y, n)=$ ( $g y, n+a(g, y)), g \in G, y \in Y_{,} n \in \mathbb{Z}$, is ergodic with respect to $\mu \times m, m$ being the counting measure on $\mathbb{Z}$. Let now $\alpha \in[0,1)$ be irrational, and consider the action of $G$ on $Y \times \mathbb{T}(\mathbb{T}=\mathbb{R} / \mathbb{Z})$ given by $g(y, t)=(g y, t+\alpha \cdot a(n, y)(\bmod 1))$ for every $y \in Y, t \in \mathbb{T}$, and $g \in G$. Since the $\mathbb{Z}$-action $(n, t) \rightarrow t+n \alpha(\bmod 1)$ on $T$ is not strongly ergodic, 
we can find a sequence $\left(B_{n}\right)$ of Borel sets in $\pi$ with $\lambda\left(B_{n}\right)=\frac{1}{2}$ for every $n(\lambda$ is the Lebesque measure on $\mathbb{T})$ and with $\lim _{n} \lambda\left(B_{n} \Delta\left(B_{n}+\operatorname{m\alpha }(\bmod 1)\right)\right)=0$ for every $m \in \mathbb{Z}$. Setting $C_{n}=Y \times B_{n}$ we obtain a non-trivial a.i. sequence for this action of $G$ on $Y \times \mathbb{T}=\mathbb{T}^{3}$. We have thus constructed a measure preserving ergodic action of $G$ on the 3-torus which is not strongly ergodic. It is well known that countable groups may have many orbitally inequivalent actions, but this example may be of interest for its simple and explicit nature. This example also leads to insight into the following phenomenon: if $G$ is amenable and $(g, x) \rightarrow g x$ a measure preserving ergodic action of $G$ on $(X, \mathscr{S}, \mu)$, then any cocycle $a$ for $G$ with values in a locally compact second countable abelian group $A$ is cohomologous to a cocycle $a^{\prime}$ taking values in a given countable dense subgroup $A^{\prime}$ of $A$. If $G$ has property $T$, if $A$ is a compact abelian group, and if $a \in Z^{1}(G, U(X, \mu, A))$ is a cocycle for $G$ which is not cohomologous to any cocycle taking values in a closed proper subgroup of $A$, then $a$ is also not cohomologous to any cocycle with countable dense range in $A$ (any such cocycle would give rise to an action of $G$ which is not strongly ergodic). Using the methods of [8] one can make this last statement stronger by specifying which dense subgroups of $A$ can occur as ranges of cocycles, but we shall not go into this.

\section{Property $T$ and boundedness of cocycles}

Let $G$ be a countable group, $(X, \mathscr{S}, \mu)$ a standard non-atomic probability space, and $(g, x) \rightarrow g x$ a measure preserving ergodic action of $G$ on $(X, \mathscr{P}, \mu)$. The following assertion was mentioned to me as a conjecture by $H$. Furstenberg. During the preparation of this paper I received a preprint by R. Zimmer containing the same result with a different proof (cf. [17]).

(4.1) Proposition. Let $A$ be a locally compact second countable amenable group. If $G$ has property $T$, and if $a \in Z^{1}(G, U(X, \mu, A))$ is a cocycle for the action of $G$ on $(X, \mathscr{S}, \mu)$, then there exists a compact subgroup $K$ of $A$ and a cocycle $a^{\prime}$ cohomologous to a taking values in $K$.

(4.2) Corollary. Let $G$ be a countable group. The following conditions are equivalent.

(1) For every measure preserving ergodic action of $G$ on $(X, \mathscr{S}, \mu)$, every locally compact second countable amenable group $A$ and every cocycle $a \in Z^{1}(G, U(x, \mu, A))$ there exists a cohomologous cocycle $a^{\prime}$ taking values in a compact subgroup of $A$.

(2) G has property $T$.

Proof. Apply proposition 4.1 and theorem 3.4 .

(4.3) Corollary. Suppose $G$ has property $T$ and $A$ is a locally compact second countable group. Let $A_{0}$ be the maximal unimodular subgroup of $A$, i.e. $A_{0}=$ $\{\alpha \in A: \Delta(\alpha)=1\}$, where $\Delta$ is the modular function of $A$. Then every cocycle a $\in$ $Z^{1}(G, U(X, \mu, A))$ is cohomologous to a cocycle taking values in $A_{0}$.

Proof. The cocycle $\Delta \cdot a \in Z^{1}\left(G, U\left(X, \mu, \mathbb{R}^{+}\right)\right)$is a coboundary. 
Turning now to the proof of proposition 4.1 and of some related results we let $A$ be a locally compact second countable group with left Haar measure and assume that $G$ is a countable group, $(X, \mathscr{Y}, \mu)$ a standard non-atomic probability space, and $(g, x) \rightarrow g x$ a measure preserving ergodic action of $G$ on $(X, \mathscr{S}, \mu)$. We put $\tilde{X}=X \times A, \tilde{\mu}=\mu \times \lambda$ and write $\tilde{\mathscr{S}}$ for the product Borel field on $\tilde{X}$. Let now $a \in Z^{1}(G, U(X, \mu, A))$, and consider the skew product action

$$
g(x, \alpha)=(g x, a(g, x) \alpha)
$$

of $G$ on $(\tilde{X}, \tilde{\mathscr{Y}}, \tilde{\mu})$, where $g \in G, x \in X, \alpha \in A . W^{a}$ will denote the unitary representation of $G$ on $L^{2}(\tilde{X}, \tilde{\mathscr{S}}, \tilde{\mu})$ given by

$$
\left(W_{\mathrm{g}}^{a} f\right)(x, \alpha)=f\left(g^{-1} x, a\left(g^{-1}, x\right) \alpha\right)
$$

for every $f \in L^{2}(\tilde{X}, \tilde{\mathscr{S}}, \tilde{\mu})$ and $(x, \alpha) \in \tilde{X}$.

(4.4) LemMA. Suppose that $W^{a}$ contains the trivial representation. Then there exists $a$ compact subgroup $A^{\prime}$ of $A$ and a cocycle $a^{\prime}$ cohomologous to a and taking values in $A^{\prime}$.

Proof. Consider the ergodic decomposition of $\tilde{\mu}$ with respect to the action (4.1) of $G$ on $(\tilde{X}, \tilde{\mathscr{S}}, \tilde{\mu})$. The existence of a $G$-invariant set $B \in \tilde{\mathscr{S}}$ with $0<\tilde{\mu}(B)<\infty$ implies that a.e. measure in the ergodic decomposition of $\tilde{\mu}$ is finite. For every $\alpha \in A,(x, \beta) \in \tilde{X}$, let $R_{\alpha}(x, \beta)=\left(x, \beta \alpha^{-1}\right)$. The action $(\alpha,(x, \beta)) \rightarrow R_{\alpha}(x, \beta)$ of $A$ on $(\tilde{X}, \tilde{\mathscr{F}}, \tilde{\mu})$ is measure preserving and commutes with the $G$-action. A standard measure theoretic argument allows us to assume that every measure $\sigma$ in the ergodic decomposition of $\tilde{\mu}$ has the following properties:

(a) $\sigma$ is finite and invariant under $G$;

(b) if $\pi_{1}: \tilde{X} \rightarrow X$ denotes projection onto the first coordinate, then $\sigma \pi_{1}^{-1}$ is a constant multiple of $\mu$ (cf. [14, theorem 1.3]);

(c) for every $\alpha \in A, \sigma R_{\alpha}^{-1}$ is either equal to $\sigma$ or singular with respect to $\sigma$. We choose and fix a measure $\sigma$ on $(\tilde{X}, \tilde{\mathscr{S}})$ satisfying $(a)-(c)$ and decompose it with respect to $\pi_{1}: \sigma=\int \sigma_{x} \mathrm{~d} \mu(x)$, where each $\sigma_{x}$ is concentrated on the set $A_{x}=$ $\{(x, \alpha): \alpha \in A\}$. We define $\sigma_{x}^{*}$ on $A$ by setting $\sigma_{x}^{*}(D)=\sigma_{x}(\{(x, \alpha): \alpha \in D\})$ for every Borel set $D \subset A$. Condition $(c)$ above, combined with the fact that the measures $\sigma_{x}^{*}$ are all finite, implies the existence of a compact subgroup $\boldsymbol{A}^{\prime}$ of $\boldsymbol{A}$ with normalized Haar measure $\lambda^{\prime}$ such that $\sigma_{x}^{*}$ is a constant multiple of a left translate of $\lambda^{\prime}$ on $A$, for $\mu$-a.e. $x \in X$. In other words we can find Borel maps $b: X \rightarrow A$ and $c: X \rightarrow \mathbb{R}^{+}$with $\sigma_{x}^{*}(B)=c(x) . \lambda^{\prime}\left(b(x) \cdot B \cap A^{\prime}\right)$ for every Borel set $B \subset A$ and for a.e. $x \in X$. We conclude that $a^{\prime}(g, x)=b(g x)^{-1} a(g, x) b(x) \in A^{\prime}$ for every $g \in G$ and for $\mu$-a.e. $x \in X$. The lemma is proved.

(4.5) LemMA. Suppose $G$ has property $T$ and $A$ is amenable. Then $W^{a}$ contains the trivial representation.

Proof. Let $\left(K_{n}\right)$ be a sequence of compact subsets of $A$ which increases to $A$, and let $\left(K_{n}^{*}\right)$ be an increasing sequence of compact sets in $A$ with

$$
\max _{\alpha \in K_{n}} \frac{\lambda\left(K_{n}^{*} \Delta \alpha K_{n}^{*}\right)}{\lambda\left(K_{n}^{*}\right)}<1 / n .
$$


We set

$$
f_{n}(x, \alpha)= \begin{cases}\lambda\left(K_{n}^{*}\right)^{-1 / 2} & \text { for } \alpha \in K_{n}^{*}, \\ 0 & \text { otherwise, }\end{cases}
$$

and observe that, for every $g \in G$,

$$
\lim _{n}\left\langle W_{\mathrm{g}}^{a} f_{n}, f_{n}\right\rangle=\int\left(W_{\mathrm{g}}^{a} f_{n}\right)(x, \alpha) \cdot f_{n}(x, \alpha) d \tilde{\mu}(x, \alpha)=1
$$

Hence the representation $W^{a}$ contains the trivial representation weakly. Property $T$ implies that $W^{a}$ contains the trivial representation. The lemma is proved.

Proposition 4.1 is now an immediate consequence of lemmas 4.4 and 4.5. Turning now to the related problem of boundedness for cocycles we recall the following definition from [9].

(4.6) Definition. Let $G$ be a countable group, $(X, \mathscr{S}, \mu)$ a standard non-atomic probability space, $(g, x) \rightarrow g x$ a measure preserving ergodic action of $G$ on $(X, \mathscr{S}, \mu)$, and $A$ a locally compact second countable group. A cocycle $a \in Z^{1}(G, U(X, \mu, A))$ is called bounded if, for every $\varepsilon>0$, there exists a compact set $K_{\varepsilon} \subset A$ with $\mu\left(\left\{x: a(g, x) \notin K_{\varepsilon}\right\}\right)<\varepsilon$ for every $g \in G$.

(4.7) TheOREM. Let $G$ be a countable group, $(g, x) \rightarrow g x$ a measure preserving ergodic action of $G$ on a standard non-atomic probability space $(X, \mathscr{P}, \mu)$, and $A$ a locally compact second countable group. A cocycle $a \in Z^{1}(G, U(X, \mu, A))$ is bounded if and only if it is cohomologous to a cocycle $a^{\prime}$ taking values in a compact subgroup $A^{\prime}$ of $A$.

Proof. Suppose $a$ is bounded and choose a compact set $K_{1} \subset A$ with $\mu\left(\left\{x: a(g, x) \notin K_{1}\right\}\right)<\frac{1}{2}$ for every $g \in G$. Let $N(e)$ be a compact neighbourhood of the identity in $A$ with $\lambda(N(e))=1$ and let $K=K_{1}^{-1} N(e)$. Put

$$
f_{1}(x, \alpha)= \begin{cases}1 & \text { if } \alpha \in N(e) \\ 0 & \text { otherwise }\end{cases}
$$

and

$$
f_{2}(x, \alpha)= \begin{cases}1 & \text { if } \alpha \in K \\ 0 & \text { otherwise }\end{cases}
$$

Clearly we have $\left\langle W_{g}^{a} f_{1}, f_{2}\right\rangle>\frac{1}{2}$ for every $g \in G$. Let $C$ be the closed convex hull of $\left\{W_{\mathrm{g}}^{a} f_{1}: g \in G\right\}$ in $L^{2}(\tilde{X}, \tilde{\mathscr{S}}, \tilde{\mu})$. Every element $\phi \in C$ satisfies $\left\langle\phi, f_{2}\right\rangle \geq \frac{1}{2}$, so that $0 \notin C$. The Ryll-Nardzewski fixed point theorem implies the existence of a function $\phi_{0} \in C$ with $W_{g}^{a} \phi_{0}=\phi_{0}$ for every $g \in G$, and we conclude that $W^{a}$ contains the trivial representation. Lemma 4.4 completes the proof of the theorem, since the converse is obvious.

(4.8) Corollary. Suppose $G$ has property $T$. Then every cocycle for $G$ with values in a locally compact second countable amenable group is bounded.

Acknowledgement. The author would like to thank the referee for pointing out an error in the original manuscript. 


\section{REFERENCES}

[1] A. Connes. A factor of type $\mathrm{II}_{1}$ with countable fundamental group. Preprint.

[2] A. Connes \& B. Weiss. Property T and almost invariant sequences. Israel J. Math. 37 (1980), 209-210.

[3] A. Connes, J. Feldman \& B. Weiss. An amenable equivalence relation is generated by a single transformation. Preprint.

[4] A. del Junco \& J. Rosenblatt. Counterexamples in ergodic theory and number theory. Math. Annalen 245 (1979), 185-197.

[5] J. Feldman \& C. C. Moore. Ergodic equivalence relations, cohomology, and von Neumann algebras. I. Trans. Amer. Math. Soc. 234 (1977), 289-324.

[6] F. P. Greenleaf. Invariant Means on Topological Groups. Van Nostrand: New York, 1969.

[7] V. Losert \& H. Rindler. Almost invariant sets. Bull. London Math. Soc. 41 (1981), 145-148.

[8] V. Losert \& K. Schmidt. A class of probability measures on groups arising from some problems in ergodic theory. In Probability Measures on Groups. Springer Lecture Notes no. 706, pp. 220-238. Springer: Berlin, 1979.

[9] C. C. Moore \& K. Schmidt. Coboundaries and homorphisms for non-singular actions and a problem of H. Helson. Proc. London Math. Soc. 40 (1980), 443-475.

[10] K. R. Parthasarathy. Probability Measures on Metric Spaces. Academic Press: New York, 1967.

[11] K. R. Parthasarathy \& K. Schmidt. On the cohomology of a hyperfinite action. Mh. Math. 84 (1977), 37-48.

[12] J. Rosenblatt. The Haar integral is the unique invariant mean for automorphisms of the torus. Preprint.

[13] K. Schmidt. Cocycles of Ergodic Transformation Groups. Macmillan (India), 1977.

[14] K. Schmidt. Unique ergodicity for quasi-invariant measures. Math. Z. 167 (1979), 169-172.

[15] K. Schmidt. Asymptotically invariant sequences and an action of SL $(2, \mathbb{Z})$ on the 2-sphere. Israel $J$. Math. 37 (1980), 193-208.

[16] R. Zimmer. Amenable ergodic group actions and an application to Poisson boundaries of random walks. J. Funct. Anal. 27 (1978), 350-372.

[17] R. Zimmer. On the cohomology of ergodic actions of semisimple Lie groups and discrete subgroups. Preprint. 